\title{
MEASUREMENT OF IN-SITU FLOW VELOCITY USING SINGLE-MOLECULE DETECTION FOR THE APPLICATION OF BIOMOLECULE QUANTIFICATION
}

\author{
Shu-Yi Chao*, Chunyang Zhang and Tza-Huei Wang \\ Mechanical Engineering Department and Whitaker Biomedical Engineering Institute, Johns Hopkins University \\ Baltimore, Maryland 21218, USA
}

\begin{abstract}
This paper describes a novel method for measuring flow velocity within a micro capillary using single-molecule detection. A custom confocal fluorescence spectroscopic system is implemented to detect fluorescent bursts of single molecules flowing through a minute laser focal volume. Durations of singlemolecule fluorescent bursts, which are found to be strongly related to the molecular transit times through the detection volume, are statistically analyzed to determine the in-situ flow speed. Low concentration $(<1 \mathrm{pM})$ of small fluorophores $(<1 \mathrm{~nm})$ can be used for analysis, rendering it a minimally invasive flow measurement technique. This single-molecule method simultaneously determines both the number of flow-through biomolecules and the associated fluid volume, and therefore can be used for quantitative analysis of biomolecules.
\end{abstract}

\section{INTRODUCTION}

The ability to detect and measure precise quantities of biomolecules, such as DNA and proteins, is of great importance to the fields of genomics, proteomics, and medical diagnostics. Quantifying the transcriptional and translational levels of specific genes and proteins is essential to studies of gene function and disease. To date, a variety of techniques have been developed for quantifying specific mRNA and proteins. Among those, polymerase chain reaction (PCR) based assays provide the most sensitive method for quantitative detection $[\mathbf{1 , 2}]$. However, the problems of contamination and variation in the amplification efficiency complicate the application of PCR for precise quantification [3]. Recent advances in confocal fluorescence spectroscopy have enabled detection of biomolecules with singlemolecule sensitivity [4-6]. Single-molecule detection (SMD) provides an opportunity to detect low-abundance of biomolecules without amplification. The concentration of biomolecules (e.g. gene expression level) can be determined according to the number of targets detected, cross-sectional area of the focused laser beam, and the flow speed at the focal region. In practice, however, precise calculation is difficult to achieve because of the uncertainty in measuring flow velocity. The actual velocity of the detected molecules cannot be simply deduced from the volumetric flow rate, since the streamwise velocity varies across the microchannel due to hydrodynamic shear (Figure 1a). This velocity depends on the position of the focused laser beam in the microchannel, which is undefined.

Several methods have been reported for probing flow velocity inside microstructures. For example, micro particle image velocimetry (micro-PIV) $[\mathbf{7 , 8}]$ was applied to measure the flow profile in a microstructure by first recording positions of many small tracer beads embedded in the fluid and then utilizing a special image cross correlation process to determine the detailed flow velocity field. One deficiency of micro-PIV is its invasiveness for both the flow field and the bioanalytical environment. The beads introduced may alter the flow condition and, more importantly, may interact with the biomolecules, incurring inaccuracy in counting of biomolecules and in measurement of flow speed. Micro flow profiling using fluorescence correlation spectroscopy (FCS) was recently reported $[\mathbf{9 , 1 0}]$. The principle of FCS is based on analysis of fluctuations in the fluorescence signal due to passage of a number of fluorescent molecules through the confocal detection volume. The flow speed at the focal point can be determined by performing autocorrelation analysis of the fluorescent variations associated with the bulk flow. Although less invasive and small fluorophores are used for determining the flow speed, the fluorescence from the imported tracing agents can interfere with the signal from the fluorescently labeled targets, adding difficulty in separating signal from the noises.

In this paper, we developed a flow measurement technique that achieves noninvasiveness by directly using the fluorescent signal from target biomolecules to determine the in-situ flow speed (Figure $1 \mathrm{~b}$ and 1c). Aside from its application to quantitative detection of biomolecules, this technique can be used for general flow profile measurement with high resolution $(<0.5 \mu \mathrm{m})$.

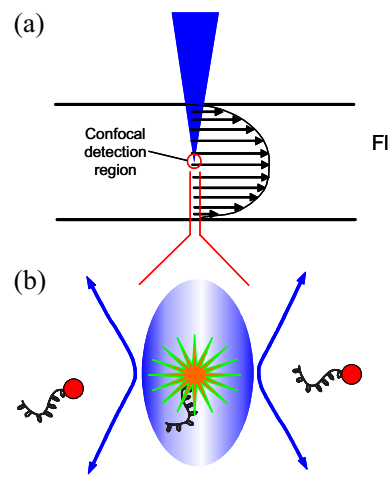

(c)

Figure 1. (a) The molecule/flow velocity within the detection volume is uncertain due to the undefined position of the confocal detection volume and the parabolic velocity profile in the microchannel. (b) A fluorescently labeled biomolecule is excited and emits fluorescence while it passes through the detection volume. (c) Molecular transit time decreases as flow velocity increases.

\section{SINGLE-MOLECULE DETECTION}

We recently achieved single-protein (human I1-2) detection using quantum dots and fluorescent colloidal nanobeads, based on confocal fluorescence spectroscopy [6]. Similarly, we accomplished detection of DNA using molecular beacon probes at the single-molecule level (Figure 2a) $[4,12]$. Molecular beacons are single-stranded, hairpin-structure oligonucleotide probes that become fluorescent only upon hybridization with their complementary target sequences [11]. We used molecular beacons

\footnotetext{
${ }^{*}$ Travel support has been generously provided by the Transducers Research Foundation and by the DARPA MEMS and DARPA BioFlips programs.
} 
as homogenous assay (separation-free) probes for detecting specific DNA sequences. Figure $2 \mathrm{~b}$ and $2 \mathrm{c}$ shows a $20-\mathrm{s}$ representative histogram of fluorescent signal of molecular beacons in the absence and presence of complementary targets. The discrete fluorescent bursts (Figure 2c) represent the events of individual fluorescent target-probe hybrids passing through the confocal detection volume. Thus, the number of targets detected can be determined according to the number of fluorescent bursts. (a)

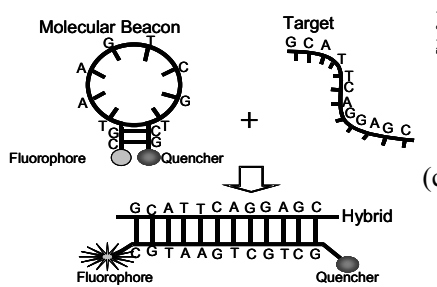

(b)

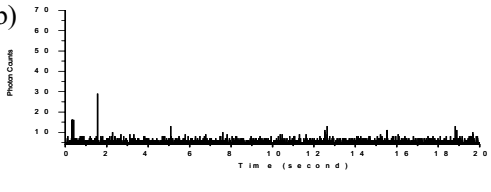

(c)

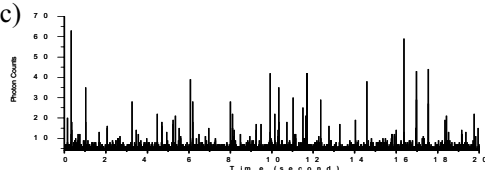

Figure 2. (a) A molecular beacon probe whose hairpin structure is destroyed when hybridized with a target, restoring fluorescence. (b) $A$ histogram of background fluorescence of molecular beacons in the absence of target DNA. (c) A histogram of single-molecule fluorescent bursts from molecular beacons-target DNA hybrids.

\section{METHOD AND PRINCIPLES}

The technique to measure the flow velocity at the focal point is based on analysis of single-molecule fluorescent histograms detected by the confocal optical apparatus. The duration of a fluorescent burst represents the transit time of a molecule passing through the detection region (Figure 1). The molecular transit time is decided by both the flow velocity at the detection point and Brownian motion of the molecule. When molecules are detected in a fast flow condition, the influence of Brownian motion on the molecular transit time can be neglected. In this regime, one would expect that increase in flow velocity results in decrease in transit time (Figure 1c). The in-situ flow velocity can then be measured through its correlation with the molecular transit times.

To better quantitatively describe this concept, an assumption is first made that the average transit time $T_{a v g}$ of a molecule passing through the probe volume is inversely proportional to the flow velocity $v$ as follows:

$$
v=\frac{k}{T_{\text {avg }}}
$$

, where $k$ is a proportionality constant. This assumption is validated through experiments described in the Results and Discussion section.

The constant $k$ is determined via a calibration process, which is performed by first measuring the flow speeds at two positions (position 1 and 2) with a known separation $S$ in the translational direction in between.

$$
v_{1}=V_{0}\left[1-\left(\frac{r_{1}}{R}\right)^{2}\right]=\frac{k}{T_{a v g_{1}}} ; v_{2}=V_{0}\left[1-\left(\frac{r_{1}+S}{R}\right)^{2}\right]=\frac{k}{T_{\text {avg } 2}}
$$

, where $R$ is the radius of the microcapillary, $r_{1}$ is the distance between the center and position 1 , and $V_{0}$ is the flow velocity at the center and is calculated according to the volumetric flow rate. After the average transit times at position 1 and position 2, $T_{\text {avg }}$ and $T_{\operatorname{avg}_{2}}$, are calculated using the measured fluorescent burst data, both of the two unknown variables, $r_{1}$ and $k$, can be determined accordingly.

\section{EXPERIMENTAL SETUP}

A schematic diagram of the custom-made single-excitation, single-emission confocal fluorescence spectroscopic system is shown in Figure 3. A $488 \mathrm{~nm}$ wavelength light beam from an aircooled argon laser (Melles Griot) is directed through a dichroic mirror and an oil immersion apochromatic objective $(100 \times$, N.A. 1.3 , Olympus) to be focused into a microcapillary $(250 \mu \mathrm{m}$ in diameter, Polymicro Technologies). A neutral density filter (1.0 o.d.) is used to attenuate the laser power. The higher wavelength emission fluorescence is collected by the same objective and passes through the dichroic mirror (Chroma Technology) which allows light of wavelength greater than $505 \mathrm{~nm}$ to pass through. A $50 \mu \mathrm{m}$ pin hole (Melles Griot) is used to suppress out-of-focus fluorescence and background noise, thus increasing the signal-tonoise ratio. The fluorescence is then filtered by a bandpass filter before detected by a single-photon avalanche photodiode (APD) (EG \& G). Samples are injected into the microcapillary through a $1000 \mu \mathrm{L}$ gastight syringe (Scientific Glass Engineering) by a highprecision syringe pump (Harvard Apparatus). A program written in LabView (National Instruments) and a digital counter (National Instruments) are used to perform data acquisition and data analysis. The excitation laser power is kept at $300 \mu \mathrm{W}$ during the experiments.

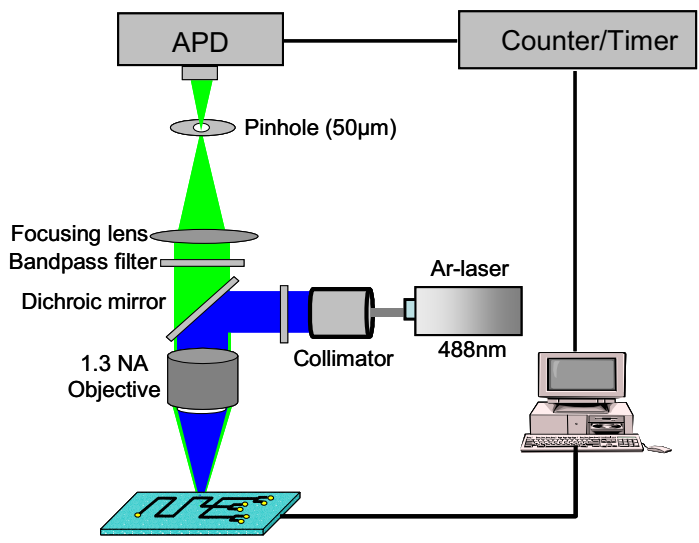

Figure 3. Schematic diagram of the single-excitation, single-emission confocal fluorescence spectroscopic system.

\section{SAMPLE PREPARATION}

We used pBR322 DNA (New England Biolabs) as target biomolecules to demonstrate the single-molecule detection-based flow measurement. A pBR322 DNA is a commonly used plasmid cloning vector in E. coli. The molecule is a double-stranded circle, 4361 base pairs in length. DNA is labeled with YOYO-1 iodide (Molecular Probes) at a ratio of 1 dye molecule/10 bp to maximize dye-DNA reaction efficiency and to minimize the noise level. YOYO-1 iodide has one carbon atom bridging the aromatic rings of the oxacyanine dye and exhibits absorption /emission maximum of 491/509 $\mathrm{nm}$ when bound to double-stranded DNA [13]. The resulting solution contained base pairs of DNA at $10^{-8} \mathrm{M}$ along with YOYO-1 at $10^{-9} \mathrm{M}$ and the reaction proceeded for $30 \mathrm{~min}$ at room temperature. The solution is further diluted 10 times with deionized water and final concentration of pBR322 DNA is $2.3 \times 10^{-13} \mathrm{M}$.

\section{RESULTS AND DISCUSSION}

Figure 4 shows a 10 -s trace (histogram) of fluorescent bursts from $\mathrm{pBR} 322$ DNA labeled with YOYO-1 flowing in a micro 

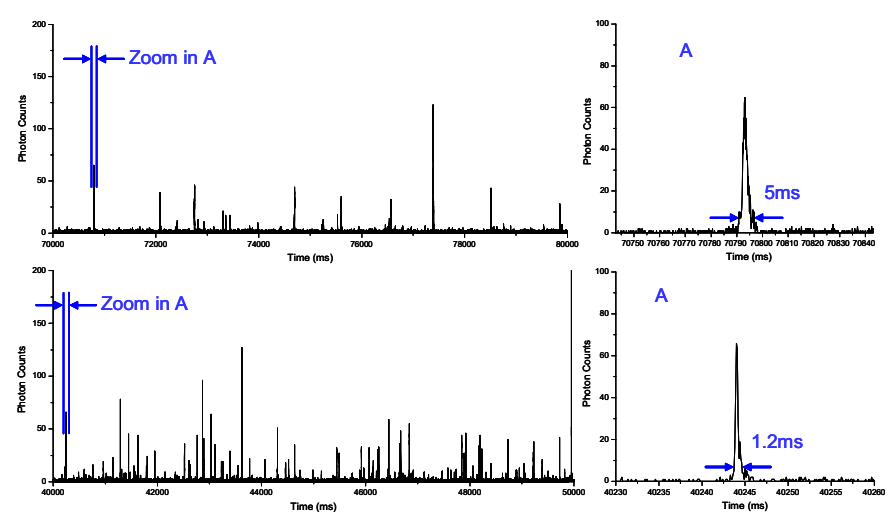

A
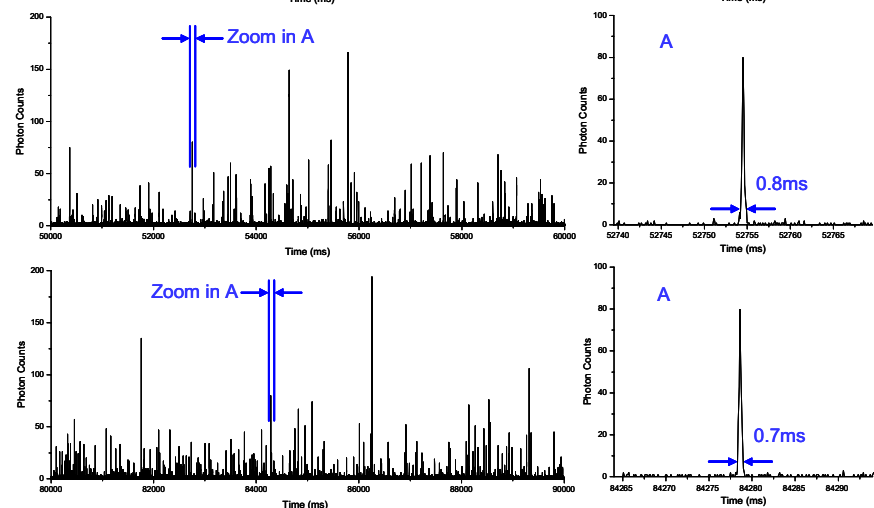

Figure 4. A 10-s trace of fluoresecent bursts histogram and a representative zoom-in of a single fluorescent burst from pBR322 DNA labeled with YOYO-1 flowing in a microcapillary (250 $\mathrm{m}$ in diameter) with different volume flow rate. The corresponding volume flow rate is $1 \mu \mathrm{L} / \mathrm{min}$, $5 \mu \mathrm{L} / \mathrm{min}, 10 \mu \mathrm{L} / \mathrm{min}$ and $15 \mu \mathrm{L} / \mathrm{min}$ from top to bottom. The bin time is $100 \mu s$.

capillary and a representative zoom-in of a single fluorescent burst for different flow conditions. The bin time (signal integration time) is $100 \mu \mathrm{s}$. Each peak represents a single DNA molecule passing through the probe volume defined by a laser beam with Gaussian intensity profile. The number of fluorescent bursts increases as the flow speed is accelerated, while the duration of a fluorescent burst decreases correspondingly.

The magnitude of a photon burst depends on the fluorescence quantum yield of the fluorescent dye, the trajectories of molecules passing through the probe region and the collection efficiency of the system [14].

$$
I(t)=Q \int_{-\infty}^{\infty} p(\vec{r}) C(\vec{r}, t) d V
$$

, where $Q$ stands for the combination of fluorescence quantum yield, the excitation cross section of fluorescence molecules, and detection quantum efficiency of detector as well as optical filtering losses in the experimental setup. $C$ is the concentration of fluorescent molecules and $p$ is the combination of the excitation intensity and collection efficiency function which can be approximated by Gaussian distribution:

$$
p(\vec{r})=I_{0} e^{-2\left(x^{2}+y^{2}\right) / w_{1}^{2}} e^{-2 z^{2} / w_{2}^{2}}
$$

, where $I_{0}$ is the excitation intensity at the center of the focused laser beam, and $w_{1}$ and $w_{2}$ are the radius and half height of the probe volume respectively, at which the intensity has dropped by a factor of $e^{-2}$. The variation of magnitude of single-DNA fluorescence bursts is attributed to the different trajectories of molecules through the non-uniform intensity within the detection volume.

When a fluorescent molecule passes exactly through the center of probe region, a burst with maximal intensity and duration is detected. In contrast, a burst with smaller intensity and duration is measured when a molecule passes through the edge of the detection volume. The probability distribution of duration of a burst is a function of the intensity distribution within the focused laser beam.

To characterize the overall transit time at the focal point, a statistical histogram of burst duration times is plotted for different flow conditions as shown in Figure 5. The background count rate is below 3 photon counts per bin in the current setup. Only bursts with a photon count rate above 8 counts per bin time are considered true events to avoid complications from the background noise. A program written in Matlab is used to determine peak duration time and perform total peak counting. The total analysis time for each measurement is 90s. As shown in Figure 5, the mean of the burst duration distribution decreases as the volume flow rate increases. In addition, the deviation and the width of the right tail in the distributions also decreases with the increase in flow rate, indicating that the influence of Brownian motion on the transit time is less significant in faster flow conditions.

The average duration is used as a characteristic transit time to determine the in-situ flow speed. Figure 6a depicts the average transit time as a function of volumetric flow rate (square). The reciprocal of the average transit time shows a linear relationship with volumetric flow rate (circle) (Figure 6b), showing that flow measurement based on average fluorescent durations is satisfactorily accurate. The plot in Figure $6 \mathrm{a}$ can be used as a standard curve to determine the molecule/flow velocity at any detection position inside the micro flow passage. The measured flow velocity starts to deviate from this linear behavior when the flow speed is smaller than $1 \mathrm{~mm} / \mathrm{s}$ because the Brownian motion effect is becoming more apparent. Therefore, for quantitative detection application, the measurement needs to be carried out in a fast flow condition in order to achieve high accuracy. A good linear relation between the number of molecules detected and
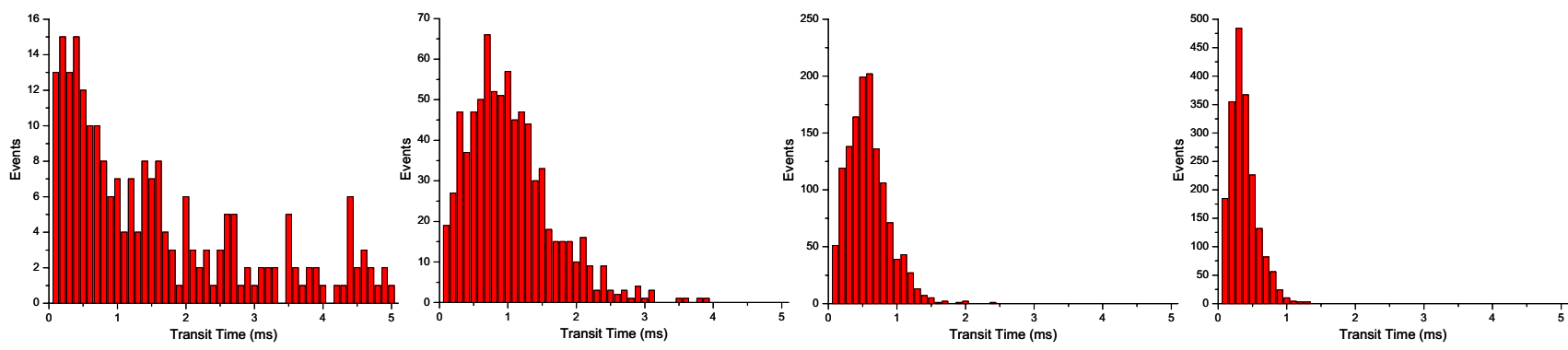


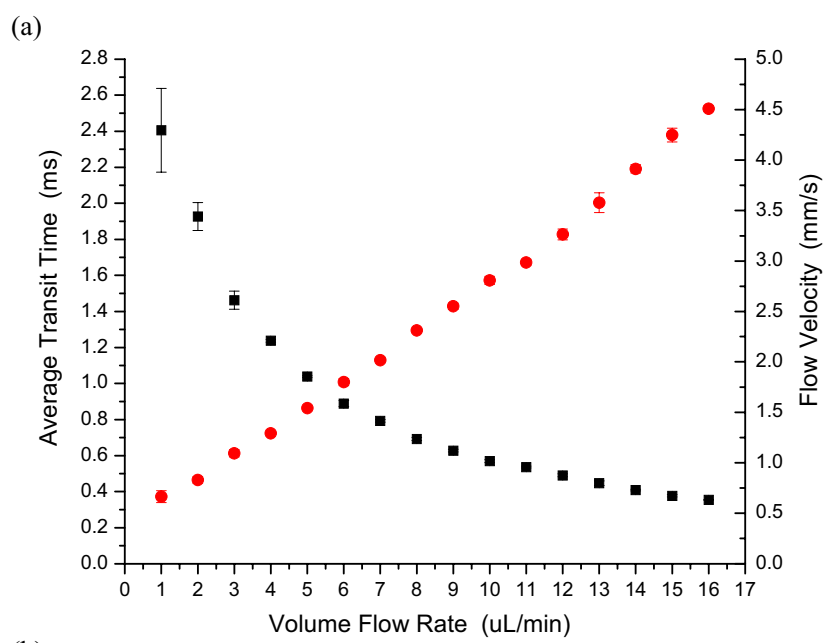

(b)

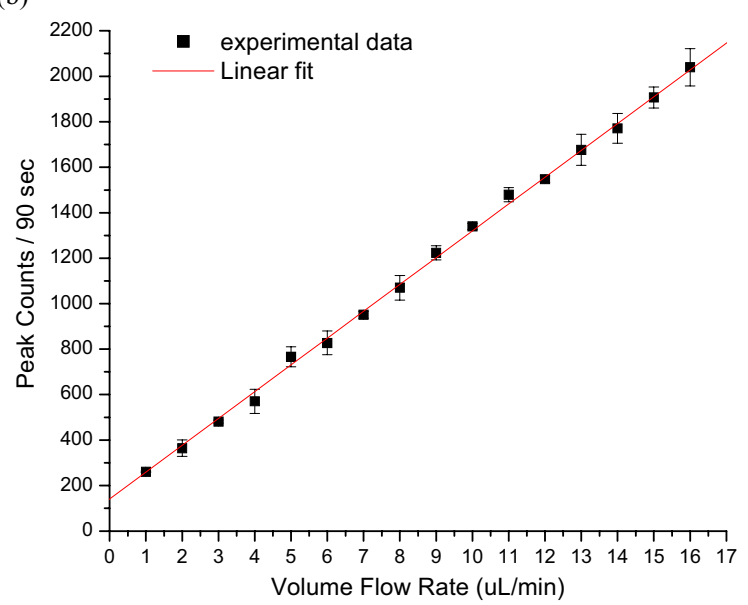

Figure 6. (a) Average transit time (square) and in-situ flow velocity (circle) as a function of volume flow rate. (b) The number of molecules detected in $90 \mathrm{sec}$ as a function of volume flow rate.

volume flow rate observed in Figure 6b demonstrates the capacity of high-reliability quantification of biomolecules using singlemolecule detection and counting in a micro flow system.

\section{CONCLUSION}

Quantification of biomolecules based on single-molecule detection in a micro flow system provides several advantages over conventional methods: extremely high sensitivity and resolution (both at the single-molecule level), requirement of low sample consumption $(<5 \mu \mathrm{L})$ and rapid analysis $(<2 \mathrm{~min})$. The development of in-situ flow velocity measurement enables accurate quantification with high reliability. We have described a generic flow measurement method which can be implemented in a variety of detection assays using different fluorescent tags. In addition, this technique can directly use the fluorescence data from the tags labeled to the molecules, without addition of extra particles to determine the flow velocity, enabling a non-invasive measurement.

\section{ACKNOWLEDGEMENTS}

This work is supported by the Whitaker Foundation. The authors thank the Transducers Research Foundation and DARPA MEMS and DARPA BioFlips programs for travel support.
1. S. A. Bustin, "Absolute Quantification of mRNA Using RealTime Reverse Transcription Polymerase Chain Reaction Assays", Journal of Molecular Endocrinology, 2000, vol 25, pp. 169-193.

2. C. T. Wittwer, M. G. Herrmann, A. A. Moss, R. P. Rasmussen, "Continuous Fluorescence Monitoring of Rapid Cycle DNA Amplification", Biotechniques, 1997, vol 22, pp. 130-138.

3. W. W. Wilke, L. D. Sutton, and R. N. Jones, "Automation of Polymerase Chain-Reaction Rests to Achieve Acceptable Contamination Rates", Clinical Chemistry, 1995, 41(4), pp. 622623.

4. T. H. Wang, S. Masset, and C. M. Ho, "A Zepto Mole DNA Microsensor", Proc. MEMS 2001, pp. 431-434.

5. T. H. Wang, P. K. Wong, and C. M. Ho, "Electrical Molecular Focusing for Laser Induced Fluorescence Based Single DNA Detection", Proc. MEMS 2002, pp. 15-18.

6. H. C. Yeh, E. Simone, C. Zhang, and T. H. Wang, "Single BioMolecule Detection with Quantum Dots in a Microchannel", Proc. MEMS 2004, pp.371-374.

7. J. G. Santiago, S. T. Wereley, C. D. Meinhart, D. J. Beebe, R. J. Adrain, "A Particle Image Velocimetry System for Microfluidics", Experiments in Fluids, 1998, vol 25, pp. 316-319.

8. S. Devasenathipathy, J. G. Santiago, S. T. Wereley, C. D. Meinhart, K. Takehara, "Particle Imaging Techniques for Microfabricated Fluidic Systems", Experiments in Fluids, 2003, vol 34, pp. 504-514.

9. M. Gösch, H. Blom, J. Holm, T. Heino, and R. Rigler, "Hydrodynamic Flow Profiling in Microchannel Structures by Single Molecule Fluorescence Correlation Spectroscopy", Analytical Chemistry, 2000, vol 72, pp. 3260-3265.

10. B. H. Kunst, A. Schots, and A. J. W. G. Visser, "Detection of Flowing Fluorescent Particles in a Microcapillary Using Fluorescence Correlation Spectroscopy", Analytical Chemistry, 2002, vol 74, pp. 5350-5357.

11. S. Tyagi, F. R. Kramer, "Molecular beacons: Probes that fluoresce upon hybridization", Nature Biotechnology, 1996, vol 14(3), pp. 303-308.

12. T. H. Wang, Y. F. Chen, S. Masset, C. M. Ho, and Y. C. Tai, "Molecular Beacon Based Micro Biological Detection System", Proc. of The International Conference on Mathematics and Engineering Techniques in Medicine and Biological Sciences (METMBS'00), Las Vegas, NV, U.S.A., 2000, pp. 295-300.

13. R. P. Haugland, "Handbook of Fluorescent Probes and Research Products", Molecular Probes Inc.

14. C. Zander, J. Enderlein, R. A. Keller, "Single Molecule Detection in Solution - Methods and Applications", Wiley-Vch, 2002.

\section{REFERENCES}

